\title{
Does the addition of concurrent visual feedback increase adherence to a home exercise program in people with stroke: a single-case series?
}

Tamina Levy ${ }^{1,2,6^{*}} \mathbb{C}$, Maria Crotty ${ }^{1,2}$, Kate Laver $^{1}$, Natasha Lannin ${ }^{3,4}$ and Maggie Killington ${ }^{1,5}$

\begin{abstract}
Objective: Evidence is accumulating for the potential benefits of technology use in stroke rehabilitation. However, few studies have examined ways in which technology can be used to increase adherence to programs after discharge from rehabilitation. The aim of this study was to determine if the addition of concurrent visual feedback, via a tablet computer, increased adherence to an exercise program following stroke. Ten participants were provided with a self-administered exercise program and were asked to perform 60 min of the exercises daily. After a baseline phase ( 1 week), participants were given a tablet computer (2 weeks) and were asked to video record each exercise session. The tablet computer was removed during the fourth week of the program.
\end{abstract}

Results: Exercise duration, measured via wrist-worn accelerometry, was investigated over the 4 weeks using the twostandard deviation (2 SD) band method. A statistically significant effect was observed in four out of ten cases, demonstrated by two successive data points occurring outside the 2 SD band during the intervention phase, suggesting that adherence was increased in response to the tablet computer use. This preliminary study indicates that the use of visual feedback, via a tablet computer, may increase adherence to an exercise program in people with stroke.

Trial registration ACTRN: ACTRN12620000252910 (26 February 2020, Retrospectively registered)

Keywords: Stroke, Technology, Feedback, Exercise

\section{Introduction}

The use of tablet devices to increase engagement in rehabilitation is increasing as services have greater access to technology [1]. Tablet computers, such as iPads ${ }^{\circledR}$, are portable and inexpensive and many individuals own these devices [2].

Whilst there is an increasing number of applications installed on tablet computers to increase participation in therapy, there is a lack of research around use of tablet

\footnotetext{
${ }^{*}$ Correspondence: tamina.levy@sa.gov.au

${ }^{6}$ Physiotherapy Department, Flinders Medical Centre -RAP Division,

Bedford Drive, Bedford Park, SA 5041, Australia

Full list of author information is available at the end of the article
}

computers as a means of video recording participation in therapy and providing real-time feedback. In a review of tablet use in stroke rehabilitation, Ameer and Ali described benefits in using a device with a camera within and outside of therapy; including allowing the therapist to record sessions and provide real-time feedback [3]. This finding is consistent with an exploratory study using video feedback of functional tasks after stroke, which found that participants who were provided with visual feedback during a task expressed greater satisfaction [4]. There is a lack of studies investigating the role real-time feedback, via tablet use, may play in promoting exercise adherence following stroke, and this should be further explored. 
Technology devices can also be utilised to monitor adherence. Adherence has been described as the extent to which a person's behaviour-taking medication, following a diet, and/or executing lifestyle changes, corresponds with agreed recommendations from a healthcare provider' [5]. Researchers and clinicians require accurate methods to measure adherence. However, measuring adherence to exercise is challenging, and consensus regarding the gold standard is lacking [6, 7]. Accelerometers are wearable sensors, designed to measure movement in activity counts [8]. The advantage of accelerometers is that an objective measure can be gained. There is evidence that accelerometers produce reliable and valid metrics of upper limb use $[9,10]$ and the feasibility of using accelerometers to monitor exercise adherence should be explored.

The primary aim of this study was to determine if the addition of concurrent visual feedback, via a tablet computer, would increase adherence to an upper limb home exercise program in people with stroke.

A secondary aim was to assess the feasibility of use of accelerometers as a method of monitoring upper limb activity during a home exercise program in people with stroke.

\section{Main text \\ Methods}

Ethical approval for this study was granted by The Southern Adelaide Clinical Human Research Ethics Committee (SAC HREC EC00188). Ten participants were recruited from the Flinders Medical Centre. Participants provided written informed consent. Inclusion criteria were diagnosis of stroke ( $>3$ months), completion of rehabilitation, and being able to pick up a small block with the strokeaffected hand. Patients were excluded if they were less than 18 years old, had a Mini-Mental State Examination score (MMSE) of less than 24 out of 30 [11], were nonEnglish speaking, or had visual deficits preventing technology use. Baseline outcome measures were collected to provide an understanding of demographics. Measures were Fugl-Meyer Assessment (FMA) [12], Modified Rankine Score (0-6) [13], Motor Activity Log-14 (MAL-14) [14], Self-Efficacy for Exercise Scale [15], Box and Block Test (BBT) [16], line bisection test [17] and the Technology Use Questionnaire (developed by authors, see Additional file 1: Appendix S1). This study adheres to the CONSORT guidelines for clinical trials (Additional file 2).

A single-case series design was employed [18] with an $\mathrm{A}-\mathrm{B}-\mathrm{A}$ design. A single-case series design allows for detailed testing of the efficacy of an intervention on a chosen outcome. In single-case experiments, sequential measurements are recorded for each participant. After an initial baseline period, an intervention is applied and the effect of this intervention relative to the baseline is investigated. Following the intervention phase, there is a follow-up phase with withdrawal of the intervention.

Participants were visited at home by the researcher. Following completion of baseline measures, the participants were instructed in the Graded Repetitive Arm Supplementary Program (GRASP) [19] and were asked to practice their program for a total of $60 \mathrm{~min}$, daily for 4 weeks. The GRASP program, a self-directed arm and hand exercise program which has been shown to improve function after stroke, prescribes $1 \mathrm{~h}$ of daily exercises and a manual of exercises is provided [19]. Participants were provided with a recording sheet to record the time spent exercising during each session.

\section{Intervention (tablet computer use)}

During the intervention phase (weeks 2 and 3), participants were provided with a tablet computer (Apple model A1474) and were asked to video record each session, using the MoviePro App, a videorecording app that was downloaded onto the tablet computer for a small fee paid by the researchers. The tablet computer was set up in front of the participant so that they were able to see themselves exercising and they were instructed to look at the screen as they exercised. Participants also recorded the start and finish times of each session on a recording sheet.

\section{Measurements}

Participants were provided with two wrist-worn accelerometers (Actigraph) and were instructed to don these prior to their exercise session and to remove them at the completion of each session. The Actigraph, a lightweight accelerometer that resembles a wristwatch, measures movement of the upper limb through acceleration.

The outcome measure used to evaluate adherence in this study was active time when wearing the accelerometer. At the completion of the study the researcher downloaded recordings from the Actigraph devices. Data was analysed using the Actilife Software, and active time was calculated for each session.

To evaluate the feasibility of accelerometry use to measure adherence, The System Usability Scale (SUS), a 1 to 5 Likert scale that measures participants' experience using technology was completed [20]. Additionally, the researcher kept a logbook, recording any issues that arose.

\section{Data analysis}

Changes in amount of activity recorded on the accelerometers from the baseline phase to the "tablet intervention" phase provided an indication of whether adherence 
to exercise increased in response to the tablet. Additionally, changes in accelerometer recorded activity following tablet removal in the follow-up phase provided extra information to inform interpretation of the data.

Following standards related to single-case series research where the participant acts as their own control, 5 measures were analysed in the baseline phase and the mean and standard deviation of measures were calculated to account for the level (mean score) and trend (slope) of the 5 measures prior to introduction of the tablet [21]. Active wear time data were then analysed using the two standard deviation (2 SD) band method which has been recommended for analysis of single-case series designs [18, 22, 23]. If two or more successive data points within the intervention phase fell outside the 2 SD band (i.e. outside the $95 \%$ confidence limits), changes from baseline to intervention were considered statistically significant. Rigour of the methodology was enhanced by replication of the design on 10 different occasions with 10 participants. This method of evaluation enables performance variability to be factored into the analysis [24].

\section{Results}

Table 1 presents the baseline demographics of participants.

\section{AIM 1: to determine if the addition of concurrent visual feedback, via a tablet computer, will increase adherence to an upper limb home exercise program in people with stroke}

Overall, a significant effect was observed in 4 out of the 10 cases (participants $1,5,7,10$ ), as demonstrated by 2 successive data points occurring outside the 2 SD band during the intervention phase, meaning that these participants performed a significantly greater amount of exercise when they were using the tablet computer. These results are represented in Fig. 1.

Furthermore, one participant (participant 9) showed a statistically significant reduction in performance at follow-up when the tablet computer was removed (Fig. 2).

Nine participants reported that they enjoyed the tablet computer and found it beneficial for giving feedback and improving engagement. Participant 1 reported that he did not like the experience as he felt like he was being watched, however he still self-reported a perception that the tablet use improved his adherence.

When time since stroke was investigated, the two participants with the longest time since their strokes (participant $1=58$ months; participant $7=110$ months), both showed a statistically significant change. Furthermore, when level of motor ability was explored, 4 of the 6 non responders to the intervention (participants 2, 3, 8 and 9) had recorded a relatively high Box and Block Test score.

\section{AIM 2: to assess the feasibility of use of upper limb} accelerometry as a method of monitoring upper limb activity The mean score for the System Usability Scale was 96.5 out of 100, indicating a high level of usability. There were several problems in terms of data collection. Issues that arose included missing data (participants 4, 9, 10); despite reportedly charging the devices, data were missing during the last three days of exercise in Participants 4 and 9. Two participants forgot to put devices on and/or off (participants 2 and 5), on one occasion for each participant. A further two participants forgot to charge the accelerometers (participants 1 and 7), and participant 7 had no recorded data after day 15 . Two participants were unable to put the device on the non-affected wrist without assistance (participants 4 and 6).

Table 1 Subject demographics at baseline

\begin{tabular}{|c|c|c|c|c|c|c|c|c|c|c|}
\hline Subject & Age & MMSE & FMA & MAL14 & Self-efficacy & MRS & BBT (affected) & $\begin{array}{l}\text { BBT (un } \\
\text { affected) }\end{array}$ & $\begin{array}{l}\text { Time } \\
\text { since stroke } \\
\text { (months) }\end{array}$ & $\begin{array}{l}\text { Time } \\
\text { since rehabilitation } \\
\text { (months) }\end{array}$ \\
\hline 1 & 64 & 26 & 63 & 7.2 & 6.4 & 2 & 16 & 29 & 58 & 20 \\
\hline 2 & 52 & 29 & 62 & 5.6 & 10.0 & 2 & 65 & 81 & 6 & 4 \\
\hline 3 & 63 & 28 & 62 & 5.6 & 8.5 & 3 & 32 & 59 & 13 & 6 \\
\hline 4 & 65 & 27 & 32 & 5.0 & 5.9 & 3 & 0 & 57 & 24 & 21 \\
\hline 5 & 65 & 30 & 62 & 4.5 & 7.4 & 3 & 19 & 63 & 14 & 9 \\
\hline 6 & 70 & 29 & 33 & 4.7 & 10.0 & 2 & 2 & 68 & 7 & 3 \\
\hline 7 & 21 & 30 & 55 & 4.7 & 6.1 & 2 & 20 & 32 & 110 & 57 \\
\hline 8 & 62 & 30 & 64 & 7.1 & 7.5 & 2 & 45 & 64 & 24 & .5 \\
\hline 9 & 56 & 30 & 65 & 7.9 & 9.7 & 2 & 48 & 54 & 3 & 1 \\
\hline 10 & 63 & 30 & 35 & 5.1 & 7.3 & 3 & 36 & 60 & 5 & 4 \\
\hline
\end{tabular}


participant 1

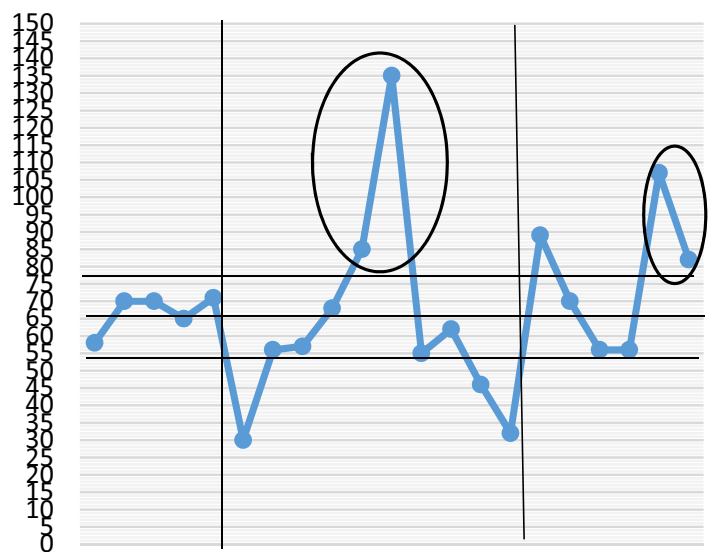

12334566789101112131415161718192021

participant 5

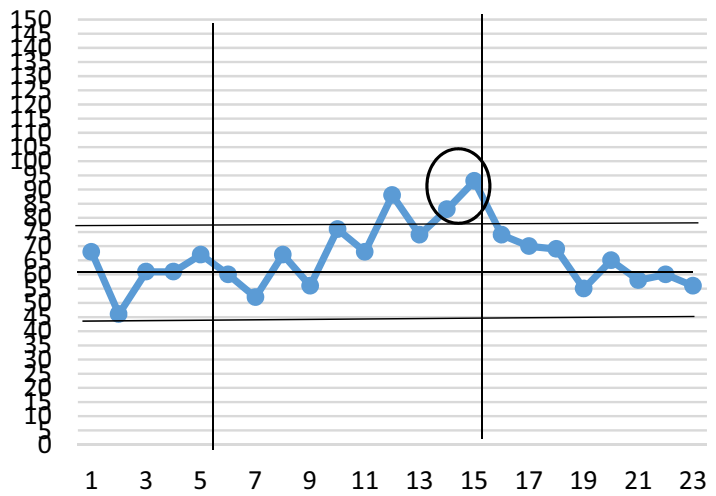

participant 7

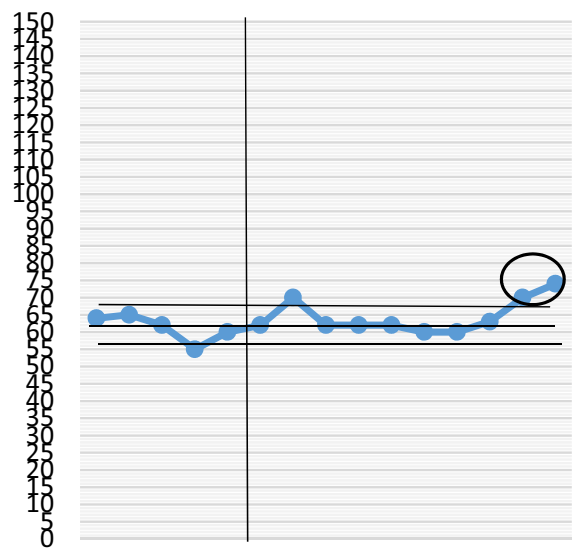

$\begin{array}{lllllllll}1 & 2 & 3 & 4 & 5 & 6 & 7 & 8 & 9 \\ 10 & 1112131415\end{array}$

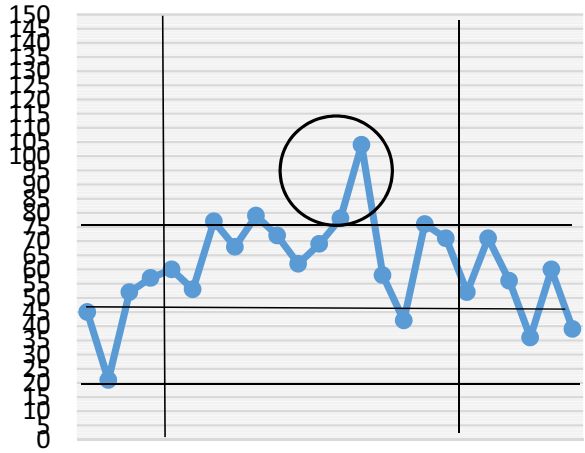

$\begin{array}{llllllllllll}1 & 3 & 5 & 7 & 9 & 11 & 13 & 15 & 17 & 19 & 21 & 23\end{array}$

Fig. 1 Data points through baseline, intervention and follow-up for Participants 1, 5, 7 and 10 (significant results circled with 2 consecutive data points outside 2 SD range). $X$ axis represents days of exercise. $Y$ axis represents exercise duration (minutes)

No issues arose with accelerometry utility or data collection in participants 3 and 8.

\section{Discussion}

This study demonstrated that using a tablet computer as a tool to promote adherence (via real-time feedback) to an upper limb home exercise program can be useful for some people with stroke. Clinicians should assess individual patient factors such as level of motivation, familiarity with technology, and level of motor impairment when considering this method of technology use. A significant improvement in the amount of exercise performed was observed in four of the 10 participants. Additionally, a further participant showed a statistically significant reduction in performance at follow-up when the tablet computer was removed.
Most participants reported positive feelings towards the approach. This is consistent with findings of Gilmore and Spaulding who reported greater satisfaction in participants who received video feedback during a functional task [4]. Furthermore, in a randomised controlled trial investigating adherence to exercise in people with stroke, Emmerson et al. [25] compared paper-based home exercises to home exercises filmed on a tablet. The authors stated that a potential benefit could be the feedback aspect of the video use and suggested that this may be evaluated further.

There were no technical issues reported with tablet computer use and all participants managed to operate the devices without any assistance or with minimal carer assistance. This aligns with qualitative data that reported 


\section{participant 9}

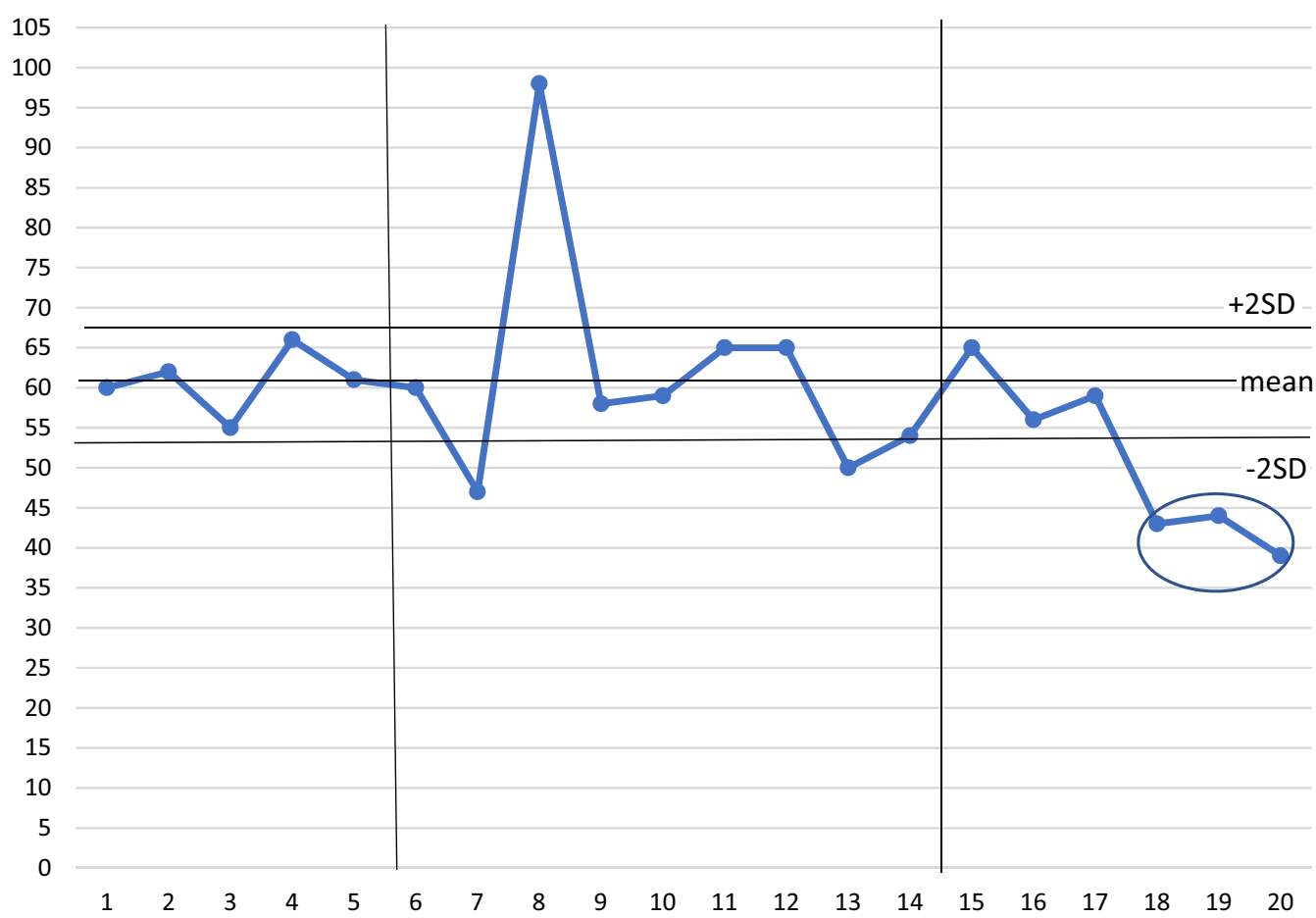

Fig. 2 Data points through baseline, intervention and follow-up for Participant 9 (significant result circled with 2 consecutive data points outside 2 SD range). $X$ axis represents days of exercise. $Y$ axis represents exercise duration (minutes)

tablet computers are easy to use, acceptable and engaging [26].

A ceiling effect was observed in participants who were highly motivated; meaning there was less 'room for improvement', and hence no statistically significant effect occurred during the intervention. Testing the effect of the intervention on the adherence of a less motivated group of participants would be valuable. Four of the participants who did not show a significant change with the intervention were those who had recorded higher scores on the Box and Block Test. It may be that the visual feedback provided by the tablet computer is more sought out, utilised and beneficial when a patient has less motor control. The two participants in this study who presented at the longest time since stroke demonstrated a significant change with the intervention. It may be that this technology is most effective when patients are in the chronic phase of recovery, but this needs to be considered with caution.

The two participants in this study who had greater motor impairment required assistance to put the accelerometers onto their non-affected wrists. Some issues with utility arose, including participants forgetting to remove and charge the accelerometers. Interventions to promote reliability of accelerometry use should be considered and may include scheduling applications and phone text reminders. This small study has demonstrated that there are issues that may reduce utility of home-based accelerometry use in people with stroke. The main advantage of using the accelerometers were that they provided accurate data on exercise time.

\section{Limitations}

The study sample was small and could be considered already motivated, having consented to a 4-week exercise program. A qualitative component would have enabled a greater exploration of participants' experiences.

\section{Supplementary information}

Supplementary information accompanies this paper at https://doi. org/10.1186/s13104-020-05202-2.

Additional file 1: Appendix S1. Technology Use Questionairre, developed by the authors.

Additional file 2. Consort checklist.

\section{Abbreviations}

GRASP: Graded Repetitive Arm Supplementary Program; SUS: System Usability Scale; 2 SD: two standard deviation band method. 


\section{Acknowledgements}

We thank all the participants who gave their time for this study.

\section{Authors' contributions}

$T L$ and MK conceived the design for the study. TL conducted the research, analysed and interpreted the data and was a major contributor in writing the manuscript. MK assisted in analysis of the data and was a major contributor in writing the manuscript. All authors read and approved the final manuscript.

\section{Funding}

This research was completed as part of TL's research higher degree studies. No external funding was received.

\section{Availability of data and materials}

The data that support the findings of this study are available on request from the corresponding author, TL. The data are not publicly available due to ethical restrictions (data may compromise the privacy of research participants).

\section{Ethics approval and consent to participate}

Participants gave written informed consent to participate in the study. Ethical approval for this study was granted by The Southern Adelaide Clinical Human Research Ethics Committee (SAC HREC EC00188).

\section{Consent for publications}

Not applicable.

\section{Competing interests}

The authors declare that they have no competing interests.

\section{Author details}

1 Flinders Health and Medical Research Institute, Flinders University, Sturt Road, Bedford Park 5042, Australia. ${ }^{2}$ Flinders Medical Centre, Rehabilitation and Palliative Services, Bedford Drive, Bedford Park, SA 5042, Australia. ${ }^{3}$ Department of Neuroscience, Central Clinical School, Monash University, Melbourne, Australia. ${ }^{4}$ Occupational Therapy Department, Alfred Health, Melbourne, Australia. ${ }^{5}$ SA Brain Injury Rehabilitation Services, Royal Adelaide Hospital, Adelaide, SA, Australia. ${ }^{6}$ Physiotherapy Department, Flinders Medical Centre -RAP Division, Bedford Drive, Bedford Park, SA 5041, Australia.

Received: 7 February 2020 Accepted: 22 July 2020

Published online: 29 July 2020

\section{References}

1. White J, et al. Tablet technology during stroke recovery: a survivor's perspective. Disabil Rehabil. 2015;37(13):1186-92.

2. Kizony $\mathrm{R}$, et al. Tablet apps and dexterity: comparison between 3 age groups and proof of concept for stroke rehabilitation. J Neurol Phys Ther. 2016;40(1):31-9.

3. Ameer K, Ali K. iPad use in stroke neuro-rehabilitation. Geriatrics. 2017;2(1):2.

4. Gilmore PE, Spaulding SJ. Motor learning and the use of videotape feedback after stroke. Top Stroke Rehab. 2007;14(5):28-36.

5. Organization WH. Adherence to long-term therapies: evidence for action. Geneva: World Health Organization; 2003. p. 2014.

6. Holden MA et al. Recommendations for exercise adherence measures in musculoskeletal settings: a systematic review and consensus meeting (protocol). Syst Rev. 2014;3(1):10.
7. Levy T, et al. A systematic review of measures of adherence to physical exercise recommendations in people with stroke. Clin Rehab. 2019:33(3):535-45.

8. Hayward KS, et al. Exploring the role of accelerometers in the measurement of real world upper-limb use after stroke. Brain Impairment. 2016;17(01):16-33.

9. Gebruers $N$, et al. Prediction of upper limb recovery, general disability, and rehabilitation status by activity measurements assessed by accelerometers or the Fugl-Meyer score in acute stroke. Am J Phys Med Rehabil. 2014;93(3):245-52.

10. Uswatte $\mathrm{G}$, et al. Objective measurement of functional upper-extremity movement using accelerometer recordings transformed with a threshold filter. Stroke. 2000;31(3):662-7.

11. Cockrell JR, Folstein MFJP. Mini-mental state examination. In: Principles and practice of geriatric psychiatry; 2002. p. 140-1.

12. Fugl-Meyer AR, et al. The post-stroke hemiplegic patient 1 a method for evaluation of physical performance. Scand J Rehabil Med. 1975;7(1):13-31.

13. Wilson $J$, et al. Improving the assessment of outcomes in stroke: use of a structured interview to assign grades on the modified Rankin Scale. Stroke. 2002;33(9):2243-6.

14. Uswatte $\mathrm{G}$, et al. Reliability and validity of the upper-extremity Motor Activity Log-14 for measuring real-world arm use. Stroke. 2005;36(11):2493-6.

15. Resnick $B$, Jenkins LS. Testing the reliability and validity of the self-efficacy for exercise scale. Nurs Res. 2000;49(3):154-9.

16. Cromwell F. Occupational therapists manual for basic skill assessment: Primary prevocational evaluation. Altadena: Fair Oaks Printing; 1976.

17. Lopes MAL, et al. Screening tests are not enough to detect hemineglect. Arq Neuropsiquiatr. 2007;65(4B):1192-5.

18. Ottenbacher KJ. Evaluating clinical change: strategies for occupational and physical therapists. New York: Williams \& Wilkins; 1986.

19. Harris JE, et al. A self-administered graded repetitive Arm supplementary program (GRASP) improves Arm function during inpatient stroke rehabilitation a multi-site randomized controlled trial. Stroke. 2009;40(6):2123-8.

20. Bangor A, Kortum PT, Miller JT. An empirical evaluation of the system usability scale. Int J Hum Computer Interact. 2008;24(6):574-94.

21. Kratochwill T, et al. Single-case designs technical documentation. 2010.

22. Crosbie $\mathrm{JH}$, et al. The adjunctive role of mental practice in the rehabilitation of the upper limb after hemiplegic stroke: a pilot studya. Clin Rehab. 2004;18(1):60-8.

23. Cashman GE, et al. Myofascial treatment for patients with acetabular labral tears: a single-subject research design study. J Orthop Sports Phys Ther. 2014;44(8):604-14.

24. Nourbakhsh MR, Ottenbacher KJJPT. The statistical analysis of singlesubject data: a comparative examination. Phys Ther. 1994;74(8): 768-76.

25. Emmerson KB, Harding KE, Taylor NF. Home exercise programmes supported by video and automated reminders compared with standard paper-based home exercise programmes in patients with stroke: A randomized controlled trial. Clin Rehab. 2016;32:9.

26. White J, et al. Tablet technology during stroke recovery: a survivor's perspective. Disabil Rehabil. 2015;37(13):1186-92.

\section{Publisher's Note}

Springer Nature remains neutral with regard to jurisdictional claims in published maps and institutional affiliations. 